\title{
309 VISUALIZING THE IMMUNOTHERAPY-INDUCED SPATIAL REORGANIZATION OF THE TUMOR-IMMUNE MICROENVIRONMENT BY CODEX MULTIPLEX IMAGING
}

${ }^{1}$ Ruan Medrano*, ${ }^{1}$ Fei Han, ${ }^{2}$ Bassem Ben Cheikh, ${ }^{3}$ Patrick Leinert, ${ }^{3}$ Wm Pat Leinert, ${ }^{2}$ Oliver Braubach, ${ }^{1}$ Robert Schreiber. 'Washington University School of Medicine, St Louis, MO, USA; ${ }^{2}$ Akoya Biosciences, Marlborough, MA, USA; ${ }^{3}$ Leinco Technologies, St. Louis, MO, USA

Background Tumors contain spatially organized microenvironments in which the location and composition of the cellular components ultimately determine tumor fate. We previously characterized CD45 + cells infiltrating T3 sarcomas using complementary forms of high-dimensional profiling (scRNAseq and CyTOF) and identified key immune cell populations that became associated either with growing tumors in mice treated with control antibody $(\mathrm{cmAb})$ or rejecting tumors in mice treated with immune-checkpoint therapy (ICT) i.e., anti-PD-1 and/or anti-CTLA-4. To better understand the effects of the intratumoral immune cell populations on one another and on the tumor itself, we used CODEX multiplex imaging to simultaneously characterize expression of 35 distinct immune cell markers on tumor tissue sections thereby maintaining the spatial relationships between effector cells and their cognate tumor cell targets

Methods T3 tumor bearing syngeneic mice were treated with $\mathrm{cmAb}$ or a-PD-1/a-CTLA-4 on days 6,9 and 12 , harvested on different days, fresh frozen, cut in $8 \mu \mathrm{M}$ sections, transferred to coverslips, and stained with a panel of commercially available and custom-made CODEX antibodies following optimized staining conditions using the manufacturer's protocol. Whole tumor tissue raw TIFF images were subjected to an image processing pipeline developed by Akoya Biosciences, Inc, where dapi+ cells used for cell segmentation by the Startdist method. Unsupervised clustering using Scanpy toolkit based on normalized marker expression profiles identified and validated 12 unique cell clusters

Results When compared to the cmAb group, a-PD-1/a-CTLA-4 tumors harvested on day 10 displayed a marked increase in percentage as well as density of CD4+ and CD8 $+\mathrm{T}$ cells, Ly6G neutrophils, MHC-II+cd11c+cd24+DCs and a decrease of CD140a+ tumor cells. Spatial analysis indicated statistically significant differences in the organization of these cell types upon treatment with a-PD-1/a-CTLA-4 compared to $\mathrm{cmAb}$ treatment. Specifically, immune cells were found at the border of the tumor in $\mathrm{cmAb}$ treated mice and heavily infiltrated tumors following ICT. Neighborhood identification analysis revealed changes in the composition of the main neighborhood clusters, of which the top three clusters present in $\mathrm{cmAb}$ treated tumors (macrophage, tumor, and other immune cells) were substituted by clusters of $\mathrm{CD} 4+$ and $\mathrm{CD} 8+\mathrm{T}$ cells, neutrophils, and macrophages.

Conclusions These results not only confirm the remodeling of both the lymphoid and myeloid compartments previously observed with scRNAseq and CyTOF but also suggest that changes in the spatial organization of immune cells are drivers of the tumor rejection process upon treatment with ICT.

http://dx.doi.org/10.1136/jitc-2021-SITC2021.309 